\title{
Electrochemical Dissolved Hydrogen Sensor Using Securely Adsorbed Platinum Particles on Nitrogen-containing Carbon Electrodes Prepared by Stepwise Electrolysis
}

\author{
Hiroaki Matsuura $^{1,2}$, Hiroyuki Kimura', Takuya Hosoda', Mai Kuwabara ${ }^{1}$ \\ ${ }^{1}$ Department of Life Science \& Green Chemistry, Faculty of Engineering, \\ Saitama Institute of Technology, 1690 Fusaiji, Fukaya, Saitama 369-0293, Japan \\ ${ }^{2}$ Department of Applied Chemistry, Graduate School of Engineering, \\ Saitama Institute of Technology, 1690 Fusaiji, Fukaya, Saitama 369-0293, Japan \\ matsuura@sit.ac.jp
}

\begin{abstract}
:
This paper describes an electrochemically activated platinum/carbon electrode incorporating nitrogen atom containing functional groups that was fabricated by stepwise electrolysis. This electrode exhibited specific electrocatalytic activity in relation to hydrogen oxidation reaction as determined by hydrodynamic voltammetry. In spite of relatively large size platinum particles, they are securely adsorbed on the activated carbon electrode compared with electrodeposited platinum on non-treated glassy carbon electrode. Moreover, we successfully developed an electrochemical sensor for dissolved hydrogen using the electrochemically activated carbon electrode modified with platinum particles as the working electrode. Although platinum particles modified $\mathrm{N}$-terminated carbon electrodes using carbon powder materials have been reported, our nitrogen-containing functional groups introduced glassy carbon electrode is first report to use solid electrode for electrodeposition of platinum particles and apply for oxidizing hydrogen molecules. The solid electrodes are more advantageous for developing hydrogen sensors because it is free standing and does not require other processes for electrode fabrication such as high temperature treatment and modification of Nafion.
\end{abstract}

Key words: Electrochemical Sensor, Dissolved Hydrogen, Stepwise Electrolysis, Electrodeposited Platinum Particles, Electrocatalytic Activity

\section{Introduction}

Carbon materials have been identified as an electrocatalytic material for the electrochemical determination of various species, and their wide variety of structurally dependent electronic and electrochemical properties [1]. In addition, the chemical modification of carbon surfaces has been used for their fields include electroanalytical chemistry and electrocatalysis [2]. Recently, it has been clarified that nitrogen atoms $(\mathrm{N})$ containing functional groups can be easily introduced onto the glassy carbon (GC) and carbon felt (CF) electrode surface by the electrode oxidation of ammonium carbamate in an aqueous medium at a highly positive electrode potential [3]. More recently, we obtained very interesting results of a novel redox waves between hydrogen ion and hydrogen molecule $\left(\mathrm{H}_{2}\right)$ appearing at highly positive potential range after the long-term electrode reduction of the $\mathrm{N}$-containing functional groups introduced carbon electrode in a strong acid electrolyte. During the electrode reduction of the electro-oxidized carbon electrode in sulfuric acid electrolyte, platinum ion dissolved from platinum wire counter electrode is electrodeposited on the surface of $\mathrm{N}$-containing functional groups introduced carbon electrode. In this paper, we describe that the electrocatalytic oxidation wave of $\mathrm{H}_{2}$ can be observed by using platinum particles modified N-terminated carbon (Pt-NtC) electrodes. The properties of the electrocatalytic activity for $\mathrm{H}_{2}$ oxidation have been investigated by employing hydrodynamic voltammetry. Moreover, we developed an electrochemical sensor of dissolved $\mathrm{H}_{2}$ using $\mathrm{Pt}-\mathrm{NtC}$ electrode as the working electrode.

\section{Experimental}

A bare GC electrode was electro-oxidized in ammonium carbamate aqueous solution at +1.1 $\mathrm{V}$ (vs. $\mathrm{Ag} / \mathrm{AgCl}$ ) for 60 minutes. Next, the 
electro-oxidized GC electrode was electroreduced in $1.0 \mathrm{M}$ sulfuric acid at $-1.0 \mathrm{~V}$ (vs. $\mathrm{Ag} / \mathrm{AgCl}$ ) for 20 hours at room temperature. While the electro-reduction of the electrooxidized GC electrode in sulfuric acid electrolyte, platinum ion dissolved from platinum wire counter electrode is electrodeposited on the electro-oxidized GC electrode surface. Amperometric measurements of $\mathrm{H}_{2}$ were performed using an electrochemical measurement apparatus based on flow injection analysis (FIA). A working Pt$\mathrm{NtC}$ electrode (6 $\mathrm{mm}$ diameter), an aqueous $\mathrm{Ag} / \mathrm{AgCl}(3 \mathrm{M} \mathrm{NaCl}$ electrolyte) reference and a platinum counter electrodes were integrated on a radial flow cell. Phosphate buffer $(0.10 \mathrm{M}, \mathrm{pH}$ 7.0) was used as the carrier solution.

\section{Results and discussion}

Fig. 1 shows the hydrodynamic voltammogram (HDV) of bubbling $\mathrm{H}_{2}$ gas in $0.10 \mathrm{M}$ phosphate buffer electrolyte $(\mathrm{pH} 7.0)$ obtained by using Pt$\mathrm{NtC}$ electrode. The electro-oxidation wave of $\mathrm{H}_{2}$ appears in the potential range from $+0.4 \mathrm{~V}$ to $-0.6 \mathrm{~V}$. We examined that no oxidation wave of hydrogen molecule was observed when the bare GC electrode was used in place of Pt-NtC electrode. Then, this result means that the electrocatalytic site for $\mathrm{H}_{2}$ is produced by multielectrolyses of GC electrode surface. Therefore, it can be recognized that the electrocatalytic site acquires its activity by the electrode oxidation at $-0.6 \mathrm{~V}$ and lose one by further electrode oxidation at $+0.4 \mathrm{~V}$. Moreover, we confirmed that the electrocatalytic activity of Pt-NtC electrode for $\mathrm{H}_{2}$ oxidation did not decrease after ultrasonication for $5 \mathrm{~min}$. This fact indicates that $\mathrm{Pt}$ particles on $\mathrm{N}$-containing functional groups are securely adsorbed.

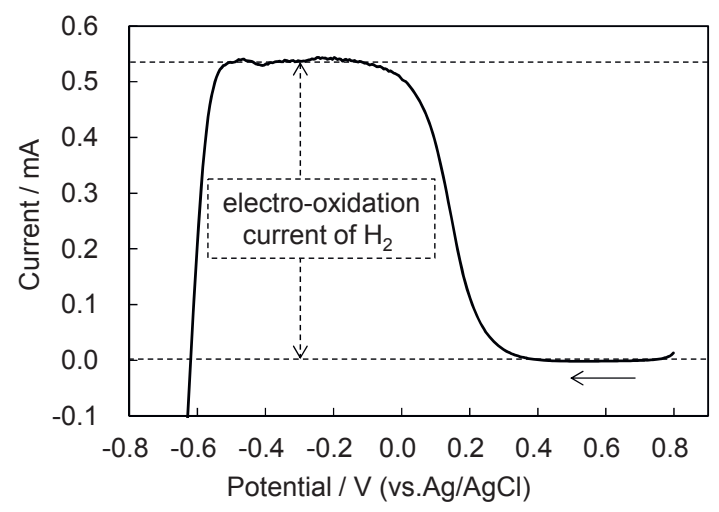

Fig. 1. $\mathrm{HDV}$ of bubbling $\mathrm{H}_{2}$ gas in $0.10 \mathrm{M}$ phosphate buffer solution ( $\mathrm{pH}$ 7.0). Bubbling rate of $\mathrm{H}_{2}: 50 \mathrm{ml} / \mathrm{min}$. Sweep rate: $50 \mathrm{mV} / \mathrm{sec}$.

We performed an electrochemical sensor based on FIA system of dissolved $\mathrm{H}_{2}$ using Pt-NtC electrode as the working electrode. The optimal applied potential to measure the peak current was measured to be $+0.05 \mathrm{~V}$ (vs. $\mathrm{Ag} / \mathrm{AgCl}$ ). When the flow rate was $5 \mathrm{ml} / \mathrm{min}$, the current

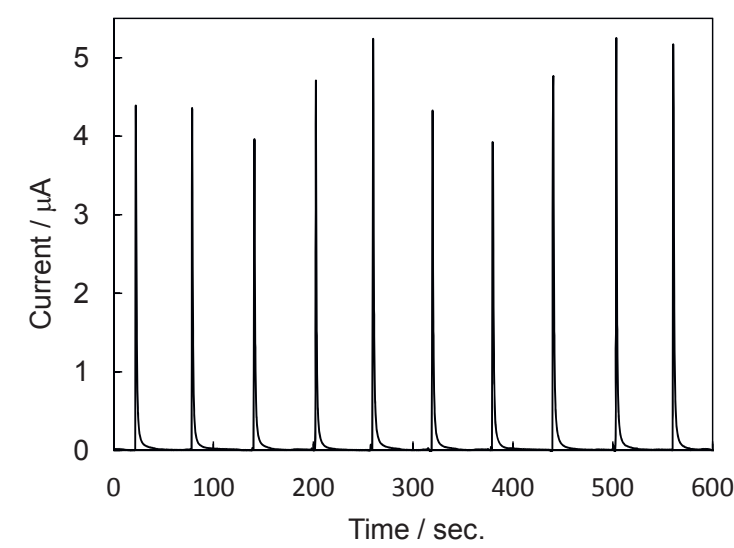

Fig. 2. Current vs. time curve obtained for the repetitive measurement of dissolved $\mathrm{H}_{2}$.

response was the maximum. In case of the optimal conditions for FIA system, typical current vs. time curve obtained by the repetitive measurement of the $\mathrm{H}_{2}$ dissolved in medium are shown in Fig. 2. This curve indicates that the measurement of dissolved $\mathrm{H}_{2}$ is finished completely in a very short time (ca. $15 \mathrm{sec}$.), and no detectable residual current fluctuation appears after the electrolysis is completed. The relative standard deviation (RSD) for tenth successive measurements was $11.8 \%$. The concentration of $\mathrm{H}_{2}$ is so unstable because the $\mathrm{H}_{2}$ dissolved in medium is gradually escaped from the solution to air, and the improvement of the stability of the concentration of dissolved $\mathrm{H}_{2}$ water is now in progress.

\section{Conclusion}

The Pt-NtC electrode was prepared by the electrode reduction in $1.0 \mathrm{M}$ sulfuric acid followed by the electrode oxidation in ammonium carbamate aqueous solution. The Pt-NtC electrode exhibited an electrocatalytic activity of $\mathrm{H}_{2}$ oxidation reaction at highly positive potential range in the hydrodynamic voltammogram. The typical current vs. time curve was obtained by the repetitive measurement of $\mathrm{H}_{2}$ dissolved in neutral medium. This result shows that the dissolved $\mathrm{H}_{2}$ concentration can be easily monitored by using $\mathrm{Pt}-\mathrm{NtC}$ electrode, and it can be expected that the electrochemical modification of carbon electrode can open the way for convenient measurement method of $\mathrm{H}_{2}$ dissolved in water.

\section{References}

[1] R. L. McCreery, Chemical Reviews, 108, 2646-2687 (2008).

[2] R. W. Murray, "Electroanalytical Chemistry", ed. A. J. Bard, Vol. 13, Marcel Dekker, New York, 191-368 (1984).

[3] S. Uchiyama, H. Watanabe, H. Yamazaki, A. Kanazawa, H. Hamana, Y. Okabe, Journal of the Electrochemical Society, 154, F31-F35 (2007). 